- Case Report

\title{
Hemiballismus in Uncontrolled Diabetes Mellitus
}

\author{
Juhaida Jaafar*, Razlina Abdul Rahman, Nani Draman, Nor Akma Yunus \\ Department of Family Medicine, Universiti Sains Malaysia, Kelantan, Malaysia
}

Hemiballismus, a subtype of chorea, is a rare movement disorder, and is most commonly found secondary to stroke. Movements are involuntary, violent, coarse, and have a wide amplitude. There is increasing report of hemiballismus occurring in non-ketotic hyperglycemia. Spontaneous improvements or remissions were observed in many patients, and treatment should be directed towards the cause of hemiballismus. There is no randomized control trial to guide clinicians in deciding the best treatment option when managing hemiballismus. Symptomatic treatment includes the use of drugs such as dopamine receptor blocker and tetrabenazine. Surgical treatment is reserved for severe, persistent, and disabling hemiballismus. This case is of an elderly woman with long standing uncontrolled diabetes who presented with abnormal movement in her left upper limb for 2 months, which resolved slowly with good control of her glucose levels. Treating physicians need to have a high index of suspicion to prevent mismanagement of the condition.

Keywords: Hemiballismus; Chorea; Choreiform Movement; Abnormal Movements; Diabetes Mellitus; Dyskinesias 


\section{INTRODUCTION}

Diabetes is a disease that impairs glucose metabolism, resulting in hyperglycemia. This chronic illness is associated with chronic inflammation and can give rise to a wide range of systemic complications, such as cardiovascular disease, neuropathy, nephropathy, retinopathy, foot damage, gastroparesis, dermopathy, and several others. Complications in the neurovascular system may involve the brain, spinal cord, cranial nerve, autonomic nervous system, or peripheral nerve. Patients with peripheral neuropathy often complain of feeling numbness, pain, or tingling sensations in their hands and feet, which can, most of the time, be easily diagnosed as a diabetes complication by the attending physician. However, this is not the same with other neurological complications, which are more complex and challenging for physicians to diagnose as part of diabetes a complication. If the neuronal part of the brain is involved, patient may present with a choreiform spectrum. Hemiballismus is one of the spectrums of choreiform, which can be a manifestation of hyperglycemia due to uncontrolled diabetes. It is rare, and its prevalence is uncertain. A case of hemiballismus in a patient with long standing uncontrolled diabetes is reported.

\section{CASE REPORT}

A 65-year-old elderly lady with underlying long-standing diabetes and hypertension presented with constant uncontrolled, purposeless and non-rhythmic movement of her left upper limb for 2 months. The abnormal movement only completely disappeared during deep sleep. About 3 weeks prior to presentation, the movement started to involve a chewing motion on left side of the face. This led to worsened and impaired basic activity of her daily living such as cooking, showering, and doing house chores. She could not identify the aggravating factors. She claimed that the condition caused tiredness, especially of the affected sites, but there was no weakness or numbness in her face and left upper limb. She denied any fever, neck pain, twitching, tremor, proximal myopathy, dysarthria, or associated slowness of speech. Her sleeping pattern and appetite were not affected.

Her medical background included long-standing diabetes mellitus and hypertension, but not with proper follow-up. Her medications included a single pill combination of metformin $500 \mathrm{mg} /$ glibenclamide $5 \mathrm{mg}$ one tablet twice daily, atenolol $100 \mathrm{mg}$ once daily, and vitamin Bcomplex once tab daily. She refused to take insulin because of her fear of needles. Furthermore, she did not comply with her treatment regime because of poor insight. Her glucometer reading was usually recorded as 'high' She also had bilateral cataracts. However, the operation was rescheduled a few times because of her uncontrolled diabetes. She denied taking any medications other than the ones prescribed.

Examination showed a smiling thin built elderly lady with a euthymic mood and appropriate affect. There was no mask-like facies. Her speech was appropriate and relevant with good articulation. Her blood pressure was 150/88 $\mathrm{mm} \mathrm{Hg}$ with a pulse rate of 100 beats per minute regular rhythm. There was no presence of carotid bruit or heart murmur. Her random capillary blood glucose was $12.0 \mathrm{mmol} / \mathrm{L}$. Urine ketone was negative.

Neurological examination showed a normal gait with good balance and absence of ataxia. No tremor and rigidity were elucidated and cerebellar signs were negative. Power, sensation, and reflexes of all four limbs were intact. There was no clonus and the Babinski sign was negative. Nonetheless, the left upper limb was moving involuntarily. The movement was wild, flailing, and repetitive with varied amplitudes and frequencies. The abnormal movement was more marked at the distal part of the limb. There was also a non-rhythmic chewing motion of the left side of her face. Cranial nerve examination was intact and not remarkable.

The case was discussed with a neurologist and based on the presented symptoms and underlying uncontrolled diabetes, diagnosis of hemiballismus secondary due to poorly controlled diabetes was suspected. She was then admitted for blood pressure and blood glucose stabilization, as well as to monitor her symptoms. Investigations in the
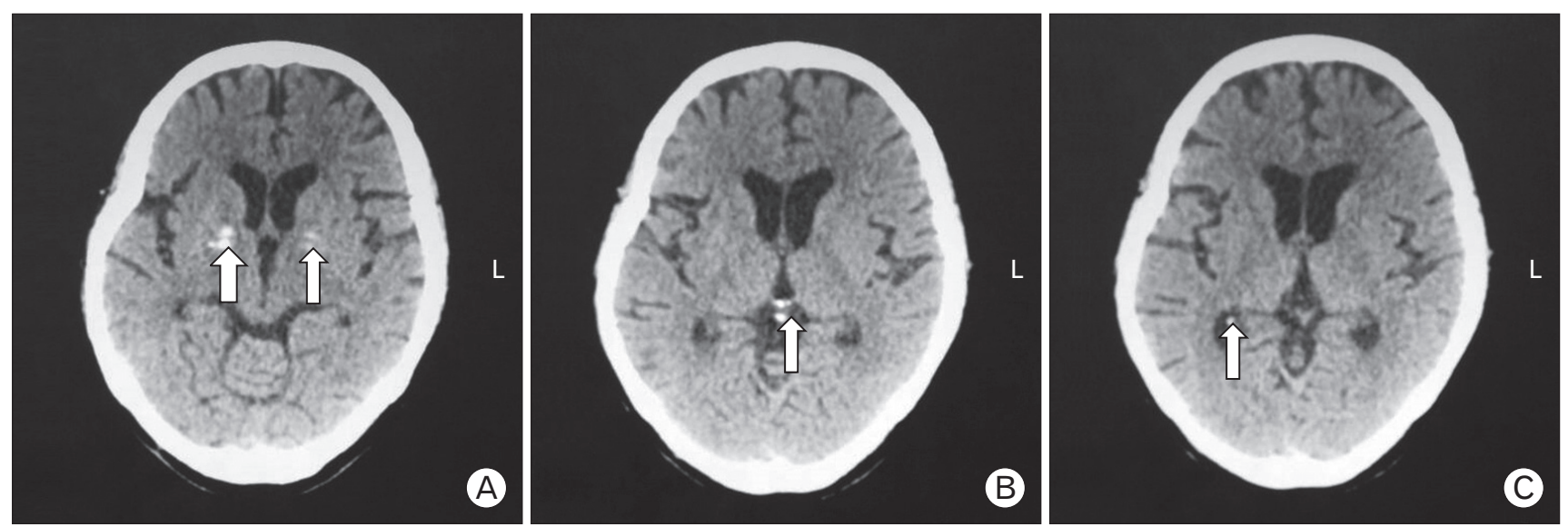

Figure 1. A computed tomography scan of the brain shows presence of physiological calcification in both basal ganglia ( $A$, arrows), pineal gland (B, arrow), and the right choroid plexus (C, arrow). 
ward showed that her hemoglobin AlC was $15.0 \%$ with hemoglobin of $11.4 \mathrm{~g} / \mathrm{dL}$. Her thyroid status was biochemically normal. Both liver and renal profiles were within the normal range. Electrocardiogram showed normal axis, sinus rhythm with T inversion at lead V3-V6. A computed tomography (CT) brain scan showed physiological calcification in both basal ganglia (Figure 1A), pineal gland (Figure 1B), and right choroid plexus (Figure 1C). The CT brain scan also showed multiple old infarct (Figure 2) with no evidence of recent infarct or hemorrhagic changes. Other acquired causes of subacute chorea such as stroke, hyperthyroidism, and electrolytes imbalance were ruled out, and a diagnosis of hemiballismus secondary to non-ketotic hyperglycemia was concluded.

Her blood glucose level improved and was within the normal range after a few days in the hospital. However, there was not much improvement in her left arm movement. Thus, oral haloperidol $2.5 \mathrm{mg}$ was added twice daily. She was then discharged with 12 units of subcutaneous premix insulin twice daily, metformin $1 \mathrm{~g}$ twice daily, atorvastatin $40 \mathrm{mg}$ once daily, aspirin $100 \mathrm{mg}$ once daily, perindopril $8 \mathrm{mg}$ once daily, and pantoprazole $40 \mathrm{mg}$ once daily.

At the 2-week follow-up, there was minimal improvement with her arm movement. However, at 4 weeks, the frequency and amplitude of left arm hemiballismus had markedly improved, together with the involuntary chewing motion. She had resumed her basic activities of daily living independently and denied any hypoglycemic symptoms. Her insulin injection technique was checked and the need to adhere to treatment regime was emphasized to both the patient and her son. Her capillary blood glucose was $4.7 \mathrm{mmol} / \mathrm{L}$ with a blood pressure of 134/75 mm Hg. Haloperidol was continued. Two months later, her abnormal movement had completely disappeared. Haloperidol was ceased.

\section{DISCUSSION}

Hemiballismus is one of the spectrums of the choreiform (abnormal involuntary movement) disorder. This abnormal movement is extremely rare. The most common cause of acquired hemiballismus is a stroke. Non-ketotic hyperglycemia is the second most common cause of this condition. ${ }^{1,2)}$ Other causes of hemiballismus are traumatic brain injury, amyotrophic lateral sclerosis, neoplasm, tuberculoma, demyelinating plaque, human immunodeficiency virus, metabolic disturbance (sodium, magnesium, calcium, hyperthyroid) and drug use (anticonvulsants, levodopa, oral contraceptives, and neuroleptic). ${ }^{1,2)}$ It is also seen more frequently in elderly females from East Asian countries, giving the possibility of genetic predisposition. ${ }^{1,2)}$

Hemiballismus was postulated to arise due to decreased activity of the subthalamic nucleus in the basal ganglia, which causes ballistic, flailing, and unwanted movements of the limbs. ${ }^{3)}$ Hemiballism that occurs in uncontrolled diabetes patient was postulated to be due to marked hyperglycemia. This extreme hyperglycemia causes hyperviscosity of the blood that eventually leads to some degree of ischemia at the basal ganglia level, ${ }^{4,5}$ leading to decreased production of GABA (gamma-aminobutyric acid) and acetylcholine at the basal ganglia, further causing disruption of normal neuronal impulse transmission and circuit. ${ }^{4,5}$ Dopaminergic activity becomes haywire causing the loss of inhibition function; hence, allowing hyperkinetic movement. The characteristic hemiballismus movements that are continuous, involuntary, irregular with variable amplitudes, and poorly patterned is caused by basal ganglia dysfunction. ${ }^{1)}$ In addition to the limbs, it can also involve facial muscles ${ }^{2)}$ which usually disappear during sleep. ${ }^{1,46)}$ Imaging of these patients may reveal abnormalities in the contralateral putamen, globus pallidus, and caudate nucleus. Magnetic resonance imaging (MRI) typically shows high signal intensity in some parts of the basal ganglia on $\mathrm{T} 1$ weighted images. ${ }^{4,6)}$
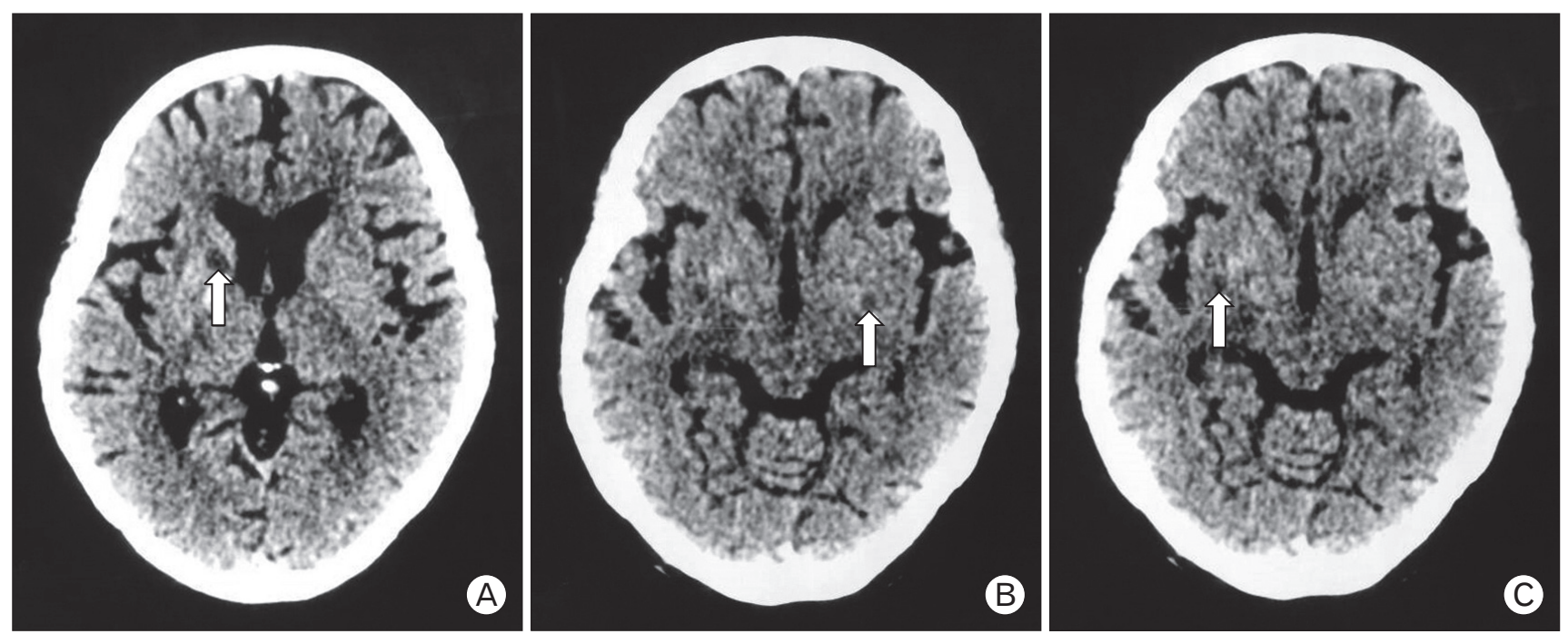

Figure 2. A computed tomography scan of the brain shows multiple hypodensities in the anterior limb of the right internal capsule (A, arrow), left basal ganglia (B, arrow), and right external capsule (C, arrow). All these well-defined hypodense lesions are in keeping with the old infarct. 
However, not all patients with hemiballismus manage to undergo extensive investigation to prove this theory due to the cost of the investigations, like MRI. In this sort of case, clinical acumen and a proper diagnostic approach may be used to arrive at the most likely diagnosis.

For this patient, a combination of clinical history and investigation excluded other common causes of hemiballismus. A brain CT was attained to exclude other potential sinister causes, such as stroke. The CT scan results, which showed physiological calcification, an old infarct (Figures 1, 2), and absence of other clinical features, was deemed enough to support the diagnosis of non-ketotic hyperglycemia as the cause of hemiballismus in this patient. Previous literature has also shown that the findings from CT scan results were used to confirm non-ketotic hyperglycemia as the cause for hemiballismus. ${ }^{7)}$ The use of MRI as a modality to confirm diagnosis is undoubtedly best; however, in certain circumstances, it is not permissible due to accessibility and cost. Although, it should not be a limiting factor for primary care doctors from making this diagnosis. Other clinical aspects from history and clinical findings may also be used to aid in the diagnosis.

Due to the elusiveness of this presentation, it can be easily missed by clinicians. Thus, clinicians must be vigilant and bear in mind that patients with long-standing poorly-controlled blood glucose levels can presents with this abnormal movement, especially among the elderly Asian. Patients need to be admitted for blood sugar normalization and to other potential causes must be excluded.5)

Usually, abnormal movements disappeared or markedly diminished once glucose level was reduced to the normal range. However, complete resolution varies by days, weeks, and months after blood glucose normalization, ${ }^{1,8)}$ and in some cases, the abnormal movements may persist for more than a year. ${ }^{2}$ In patients with a slow recovery, a trial of pharmacological treatment can be administered. Medications such as tetrabenazine, anticonvulsants, and botulinum injection trials showed mix results. Haloperidol is a common medication used, but the outcomes vary, with some patients not showing significant improvement. ${ }^{6}$ For this patient, haloperidol seemed to have a positive result. However, blood glucose normalization and time may also have contributed to improvement in the hemiballismus.

For refractory hemiballismus and disabling hemiballismus, surgical modalities such as stereotactic pallidotomy ${ }^{9}$ and deep brain stimulation can be offered in a few selected patients. ${ }^{10)}$

Even though this hemiballismus secondary to hyperglycemia is benign and usually has an excellent prognosis, this illness had great impact on the patient and her family members. Because of this abnormal movement, the patient felt handicapped and distressed, as most of her basic activity took much longer to complete and she required help from other family members. It also took a heavy toll on the family. Hence, clinicians should be tactful in tackling the biopsychosocial aspect of the patient.
This case illustrates that uncontrolled diabetes can present as a rare dyskinesia problem. Unfamiliarity with these conditions may lead the treating physician to attribute it to psychological or psychiatric disturbances. A high index of suspicion is warranted for all primary care doctors dealing with this common medical problem.

\section{CONFLICT OF INTEREST}

No potential conflict of interest relevant to this article was reported.

\section{ACKNOWLEDGMENTS}

We would like to thank Nor Diana Borian, MD (UKM) from the Department of Radiology, Universiti Sains Malaysia for her assistance with the images and the patient for giving her consent for this case report.

\section{REFERENCES}

1. Priola AM, Gned D, Veltri A, Priola SM. Case 204: nonketotic hyperglycemia-induced hemiballism-hemichorea. Radiology 2014;271:304-8.

2. Oksana S, Manon B. Overview of chorea: metabolic and endocrine disorder [Internet]. Waltham (MA): UpToDate Inc.; 2016 [cited 2017 Jul 5]. Available from: http://www.uptodate.com.

3. Pramod K. What causes hemiballismus and how can it be treated [Internet]. Tampa (FL): ePainAssist; 2017 [cited 2017 Aug 25]. Available from: https://www.epainassist.com/movement-disorders/hemiballismus.

4. Padmanabhan S, Zagami AS, Poynten AM. A case of hemichoreahemiballismus due to nonketotic hyperglycemia. Diabetes Care 2013;36:e55-6.

5. Borensztein A, Walker RH, Schell R, Guber HA. Hyperglycemia-induced involuntary movements: 2 case reports and a review of the literature. AACE Clin Case Rep 2015;1:e165-9.

6. Block H, Scozzafava J, Ahmed SN, Kalra S. Uncontrollable movements in patient with diabetes mellitus. CMAJ 2006;175:871.

7. Langer FW, Suertegaray G, Dos Santos D, Alves GR, Haygert CJ. Hemichorea-hemiballism: the role of imaging in diagnosing an unusual disorder in patients with nonketotic hyperglycemia. Radiol Bras 2016;49:267-8.

8. Barton B, Zauber SE, Goetz CG. Movement disorders caused by medical disease. Semin Neurol 2009;29:97-110.

9. Slavin KV, Baumann TK, Burchiel KJ. Treatment of hemiballismus with stereotactic pallidotomy: case report and review of the literature. Neurosurg Focus 2004;17:E7.

10. Stephanie MV, Stephen AB, Francisco T, Selim RB, Stephen TG. Chorea in adults treatment \& management [Internet]. New York (NY): Medscape; 2014 [cited 2017 Jul 5]. Available from: http://emedicine. medscape.com/article/1149854-treatment\#showall. 